\title{
lingvis.io - A Linguistic Visual Analytics Framework
}

\author{
Mennatallah El-Assady ${ }^{1}$, Wolfgang Jentner ${ }^{1}$, Fabian Sperrle ${ }^{1}$, \\ Rita Sevastjanova ${ }^{1}$, Annette Hautli-Janisz ${ }^{2}$, Miriam Butt ${ }^{2}$, and Daniel Keim ${ }^{1}$ \\ ${ }^{1}$ Department of Computer Science, University of Konstanz, Germany \\ ${ }^{2}$ Department of Linguistics, University of Konstanz, Germany
}

\begin{abstract}
We present a modular framework for the rapidprototyping of linguistic, web-based, visual analytics applications. Our framework gives developers access to a rich set of machine learning and natural language processing steps, through encapsulating them into microservices and combining them into a computational pipeline. This processing pipeline is auto-configured based on the requirements of the visualization front-end, making the linguistic processing and visualization design detached, independent development tasks. This paper describes the constellation and modality of our framework, which continues to support the efficient development of various humanin-the-loop, linguistic visual analytics research techniques and applications.
\end{abstract}

\section{Introduction}

Research at the intersection of computational linguistics, visual analytics, and explainable machine learning, is a vibrant, interesting field that broadens the horizons of all disciplines involved. Over the last years, a team of computer scientists, linguists, as well as social scientists from different areas, at the University of Konstanz, have come together to push their disciplinary boundaries through collaborative research. This collaboration resulted in the development of several mixed-initiative visual analyitcs approaches, ranging from generating high-level corpus overviews using Lexical Episode Plots (Gold et al., 2015) to sophisticated human-in-the-loop topic refinement techniques (El-Assady et al., 2018b, 2019).

This effort has helped establish the subarea of Linguistic Visualization (short: LingVis) research (Butt et al., 2019). Within this subarea, application topics we worked on include content analysis, e.g., NEREx (El-Assady et al., 2017b); discourse analysis, e.g., ThreadReconstructor (El-
Assady et al., 2018a); language change, e.g., HistoBankVis (Schätzle et al., 2017) or COHA Vis (Schneider et al., 2017); readability analysis, e.g., literature fingerprinting (Oelke et al., 2012); language modeling, e.g., LTMA (El-Assady et al., 2018c); argumentation analysis, e.g., ConToVi (ElAssady et al., 2016); explainable machine leraning, e.g., verbalization and active learning (Sevastjanova et al., 2018a,b); interactive model refinement, e.g., SpecEx (Sperrle et al., 2018); multi-corpora analysis, e.g., Alignment Vis (Jentner et al., 2017); modeling of speech features, e.g., SOMFlow (Sacha et al., 2018).

To make our linguistic visualization techniques accessible to a wider public, we strive to implement them as web-based applications. However, this is only possible on a larger scale using a framework architecture that accommodates the needs for rapid-prototyping, disguising the involved engineering complexity for application developers. Hence, we established the lingvis.io framework as a common platform, facilitating the share and reuse of implementation components. A prominent application powered by our framework is VisArgue (El-Assady et al., 2017a), an approach for multi-party discourse analysis.

In this paper, we report on our shared framework and infrastructure that drives a multitude of linguistic visualization projects, as depicted in Figure 1. The core of our framework is a flexible pipeline with automatic dependency resolution that enables application developers to request natural language processing (NLP) steps for their visualizations, which, in turn, are auto-configured based on user-defined parameters. These are chosen in a user interface that is designed to enable experts and non-experts, alike, to adapt the NLP processing to their tasks and data. The results of this processing are closely intertwined with the interactive visual analytics components, enabling, 


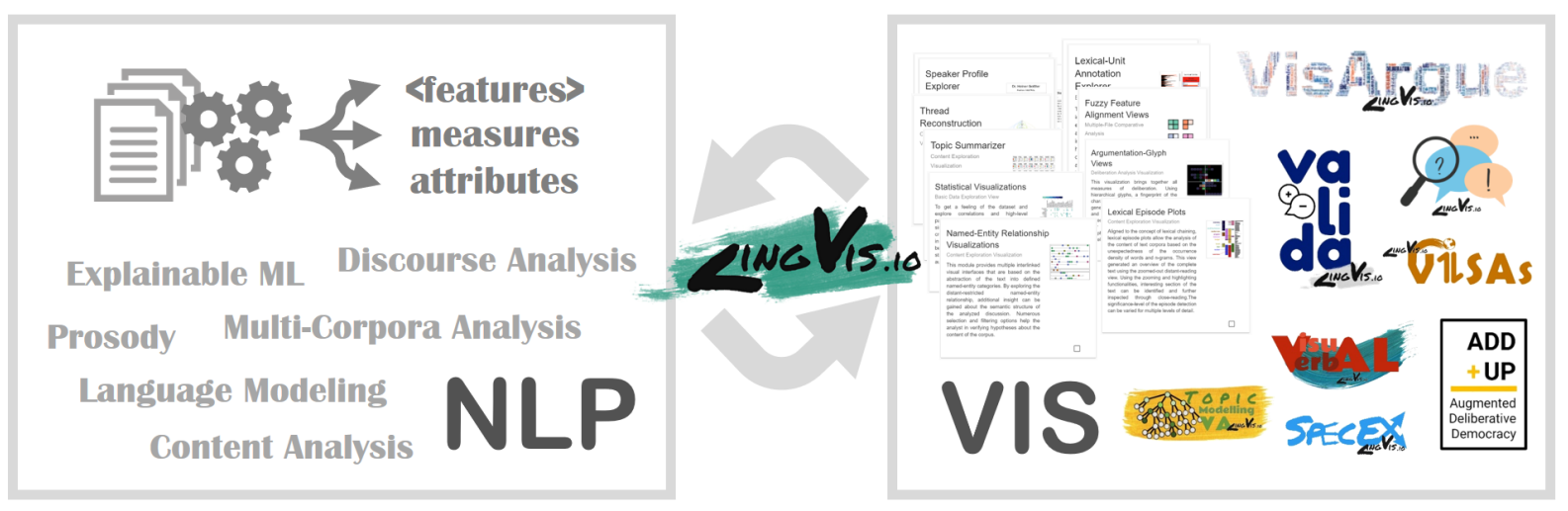

Figure 1: The lingvis.io framework driving various linguistic visualization projects based on rich NLP pipelines.

for instance, visual debugging for linguists, or insights for domain experts, such as writers, political scientists, etc. To address the trade-offs between tailored and expressive interface design, rapidprototyping, and processing flexibility, our framework architecture strictly separates and modularizes tasks into atomic components that are compartmentalized in subdomains (i.e., auto-scaling cluster environments). Developers work on their designated feature branches and efficiently test their prototypes through continuous deployment.

Related Work - Other notable frameworks related to ours include Stanford CoreNLP ${ }^{1}$, GATE $^{2}$ and Weblicht ${ }^{3}$. Facebook has recently released a deep-learning based framework for various NLP tasks, called pytext ${ }^{4}$. While they provide state-ofthe-art models, they are code-only platforms that require developers to write processing pipelines from scratch every time. More general (deep learning) frameworks, including tensorflow ${ }^{5}$ and pytorch $^{6}$ can also be used for text processing or to generate rich feature vectors like sentence-

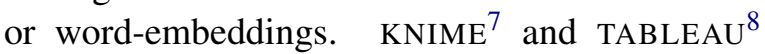
are platforms for intuitively creating data science workflows with reusable components, but are not tailored to NLP tasks specifically. While we can communicate with those frameworks through APIs to enrich our own NLP pipeline, these toolkits are solely tailored to linguistic analysis and offer no, or very limited, visualizations possibilities.

\footnotetext{
${ }^{1}$ stanfordnlp.github.io/CoreNLP

2 gate.ac.uk

3 weblicht.sfs.uni-tuebingen.de

4 github.com/facebookresearch/pytext

5 tensorflow.org

${ }^{6}$ pytorch.org

7 knime.com

8 tableau.com
}

\section{Auto-Configured Processing Pipeline}

Our framework is based on the assumption that the individual processing steps can and should be atomic in their nature. Each step holds a welldefined list of dependencies which the respective step requires to execute its task successfully. This allows us to model a processing pipeline for a given type of data input as an acyclic graph which can be processed in parallel. For example, as shown in Figure 2, to retrieve the result of a topic model, the visualization requests one or more models. Based on their dependencies to other steps, a pipeline is generated (that takes into account all user-defined parameters). Here, the topic modeling is based on descriptor vectors extracted for each document in the corpus, as well as word embedding results.

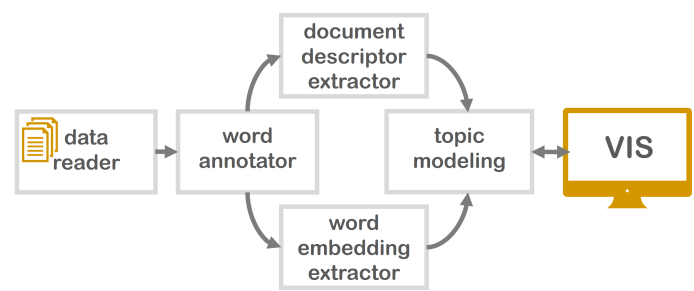

Figure 2: Dependency graph for topic model relations.

A successful implementation requires a consistent, flexible, and well-defined data model such that each step can use its transformation capabilities to semantically enrich the data. We therefore do not allow any step to modify or delete data but each step can further add metadata. This section describes the modeling of processing steps in our pipeline, as well as the underlying data structure.

\subsection{Processing Steps}

Our framework allows for progressive steps where intermediate results can be investigated and fur- 
ther steered by the user. As shown in Figure 3, in the user interface (UI), users first upload and select the data they want to process. Based on their intended tasks they then select suitable visualization components. Internally every visualization defines a list of atomic processing steps as dependencies that need to run in order to generate the desired information to visualize. In addition to a list of dependencies, visualizations define one or multiple controller endpoints. These serve as communication medium between the processing steps and the UI, and are characterized by the fact that they do not further enrich the data and cannot be defined as dependencies by any other processing step. This implies that visualization steps terminate the acyclic graph and, thus, the resulting processing pipeline.

For the initial processing, a controller in the streaming-control-layer handles the communication with every specific processing step and provides the parameter-configuration interface to the UI. This enables users to parameterize the processing for increasing flexibility. For example, a POS tagger step can be parameterized with different tagger models. Such a tagset does not need to be static but can depend on a language or be based on a user's selection. It is only constrained by the necessity of having a standardized tag set, as later steps use these tags to further process the data.

The endpoints in the two control layers separate static (default) from streaming controllers. In the default case, controllers are used to communicate the results of a completed processing step to the visualization. Streaming controllers, on the other hand, intercept a processing step while it is running to support direct user interactions. Here, progressive visualizations are shown while the respective processing step is running. The users can, therefore, directly observe, adapt, and refine the underlying machine learning models. This enables the design of tightly-coupled, human-in-the-loop interfaces for interactive model refinement and explainable machine learning.

\subsection{Data Structure Modeling}

We represent a corpus hierarchy as a recursively stacked data structure consisting of, so-called, 'document objects'. These are a modular abstraction of all levels of the hierarchy, including corpora, documents, paragraphs, sentences, etc. The highest level of our data structure consists of a

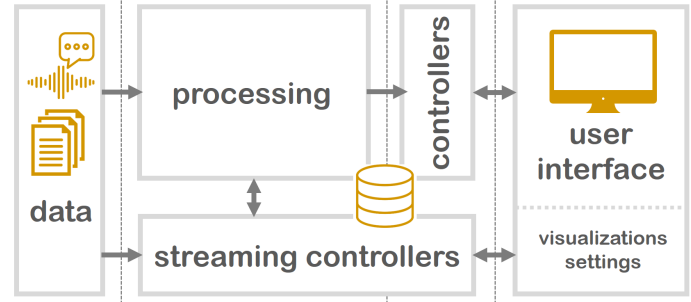

Figure 3: Schematic overview of the framework.

collection of document objects that typically represents all analyzed corpora, whereas the lowest level are single word tokens. Hence, from an ordered list of all corpora, we can descend the data structure to find a list of documents for each corpus, all the way to sub-sentence structures, multiword objects, and finally words consisting of an ordered list of tokens. This flexible data structure allows us to model arbitrary complex object hierarchies, with each object level containing an ordered list of the objects on the next level, while tokens define the terminal level.

Each processing step in the pipeline has access to the full data hierarchy. Throughout the processing, steps append additional data elements to the hierarchy objects to enrich them with computational results and metadata, making their processing results accessible to other steps downstream. Hence, through defining the pipeline dependencies, processing steps can request input data that is provided by its previous steps through defined formats, which ensures atomicity and encapsulation. This appended data is independent of a step's sophistication, which can range from simple wordlist lookups to complex deep neural network models. In the following, we describe the three data formats that can be appended to document objects.

(1) Weighted Feature Vectors (FV) - One of the key structures attached to document objects are feature vectors. These represent the transformation of text from a semi-structured data source to a high-dimensional feature space. Feature vectors represent the discretized elements of the text, often weighted descriptors extracted from the underlying text. These vectors are defined by a global signature vector that prescribes an ordered reference for the numerical weights contained in individual FVs for each document object. For example, to build a frequency-based bag-of-words model, we enable users to choose from a set of token-classes including POS-tags, named entities, lexical chains, n-grams, stop-words, etc. These are 


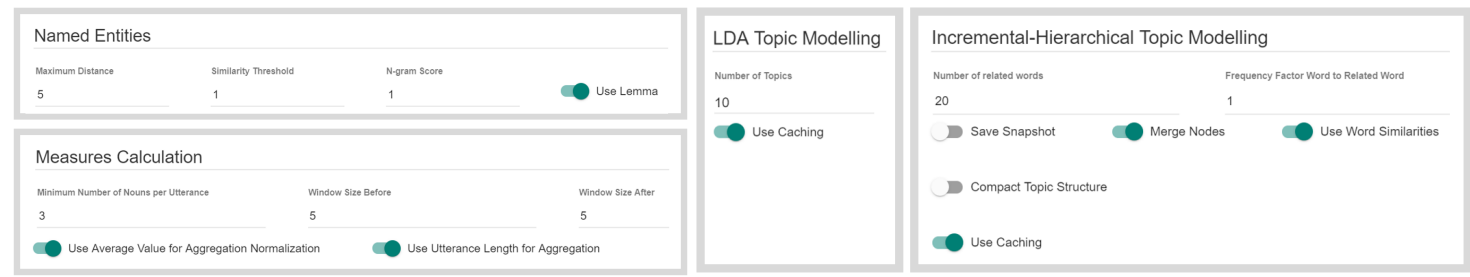

(a) Named Entity and Measure Settings UI

(b) Topic Modeling Settings UI

Figure 4: UI components for parameterizing processing steps. Available settings depend on the underlying models.

scored based on several weighting schemes, such as $\mathrm{tf}$-idf, ttf-idf, log-likelihood ratio, and other metrics, as described by El-Assady et al. (2018b). Such weighted feature vectors can describe the importance of keywords on a global level (e.g., for all analyzed corpora) or on an individual object level (e.g., for a single document). Other types of feature vectors include ones extracted using word- or sentence-embeddings, as well as vectors based on linguistic annotation pipelines.

(2) Attributes (A) - As opposed to numeric feature vectors, attributes consist of labels or pointers attached during processing. Both of these attribute types can be used to aggregate feature vectors or measures in the data hierarchy. For example, for dynamically computing all measure values related to a particular speaker and topic in a conversation, these attributes are utilized.

Labels (L) can be single flags, such as POS tags, or could consist of n-tuples, for example, to inform the types of arguments contained in the underlying text. To accommodate for labels that describe only parts of a hierarchy, we also feature window labels. These are stored in the hierarchy level above the targeted level and contain a beginning and ending indices of the children. For example, the sentence hierarchy level may contain a label consensus with a beginning index of 0 and ending index of 6 , pointing to a sub-sentence structure that encodes that the first six tokens of that sentence are indicating a consensus.

Pointers (P), on the other hand, are attributes that point to external structures, such as topics, speakers, or other entities. Such structures are usually modeled by specific processing steps and contain descriptive features of the elements they represent. For example, a topic might contain a list of descriptive keywords, whereas a speaker object would contain metadata and biography information on a speaker.

(3) Measures (M) - As a pendant to nominal attributes, measures are numeric or boolean values attached to the document objects. These are used to describe linguistic features of various types. As with the labels, a measure consists of a class name and a singular value. They are typically used to qualify properties of objects and, thus, can be aggregated through the data hierarchy. In addition, measures can be normalized, for example, based on the number of tokens in a document. We distinguish three types of measures: Boolean; numeric continuous; and numeric bi-polar. Such measures can be extracted through a variety of processing steps, ranging from simple word-list-based taggers, statistical analysis steps, rule-based annotators, through sophisticated machine learning based measure calculators. We use such measures to extract semantically relevant information or to monitor the quality of document objects with respect to selected criteria. Hence, such measures inform the visual analytics methods and expand the dimensionality of the underlying objects.

\section{User Interface}

"Simplicity comes at the cost of flexibility" (Jentner et al., 2018). The dependency-based processing model automatizes many decisions a user has to take in other frameworks. However, in order to allow domain-experts to use their knowledge and influence the underlying models, parameterization is necessary. We run a linearization of the acyclic graph prior to executing the pipeline. This allows us to display the steps and their parameters in the order of the processing-flow to support the users in their parameter estimation. To further support users we deploy guidance in the form of information pop-ups and built-in tutorials. This includes explaining how a respective processing step transforms the data and the value it adds to the task, but furthermore involves descriptions of the parameters and their estimated impact.

To exemplify this process, we describe a partial pipeline that is commonly used in our frame- 
work to demonstrate its expressiveness and flexibility. Let this partial pipeline be: (1) Named Entity Recognizer $(A-L) \rightarrow(2)$ Document Feature Extractor $(F V) \rightarrow(3)$ Topic Modeling $(A-P) \rightarrow$ (4) Measure Calculator (M). The (1) NER step labels (A-L) tokens with Named Entities. As shown in Figure $4 \mathrm{a}$, the user can define parameters such as the minimum distance and similarity score. The (2) DFE creates feature vectors (FV) on all data hierarchies. Based on the data and task, the user selects and weights the features and selects an appropriate scoring scheme. In the (3) TM step, the user selects one or multiple of the available topic models (e.g., LDA, IHTM) and parameterizes them, for example, with the number of desired topics (Figure 4b). Note that this step uses only the feature vectors extracted in the previous step. It assigns additional pointer attributes (A-P) for each document reflecting their probability to belong to a certain topic. The (4) MC then uses, for example, the topic labels to calculate measures (M) such as Topic Shift where the topic of discussion is changed within a document, or Topic Persistence where a given topic continues to be pursued by the author or speaker.

Such a pipeline is part of multiple visualization creation cycles. For example, we utilize the results of topic modeling to analyze the dynamics of speakers in a conversation transcript in ConToVi (El-Assady et al., 2016). Hence, to build such visualization approaches, we rely on the autoconfiguration of the processing pipeline, as well as the familiarity of users with their analyzed data and tasks, enabling application developers to focus on their encapsulated implementation environment without worrying about the complexity of the underlying linguisic processing.

\section{Microservice Architecture}

The modularity of our framework and atomicity of the steps is further emphasized by the use of microservices (Figure 5, s1, s2, s3). A microservice is a small, single-purpose service that exposes an API. Because our microservices are dockerized, ${ }^{9}$ the microservice itself is independent of any programming language and environment which provides us with great flexibility. Additionally, individual microservices are easier to maintain than a large, monolithic framework. An example microservice from our framework returns POS tags

\footnotetext{
${ }^{9}$ docker.com
}

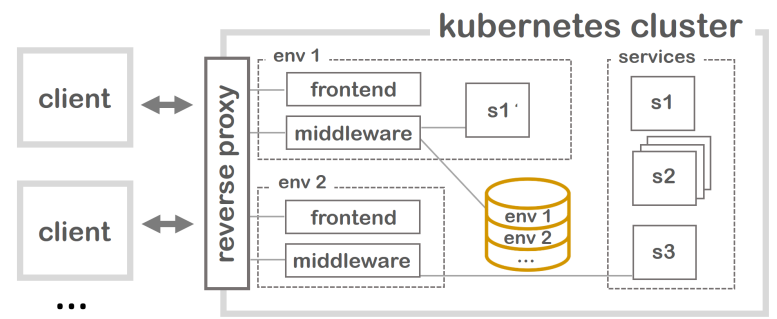

Figure 5: Multiple environments (env1, env2) of the lingvis.io framework are managed in a kubernetes cluster. Microservices $(s 1, s 2, s 3)$ are tailored to a specific task and resemble different steps of the pipeline. Any microservice can be redefined in a specific environment if variations of the functionality are needed.

for tokenized texts. Requests to that service contain a list of tokens, and are parametrized with the tagger model to use. The middleware handles the user authentication, the (processed) data, the pipeline steps, and the controllers (Figure 3). In addition, it coordinates the microservices and handles communication with their respective APIs to obtain results to add to the data.

Our framework lives in a kubernetes cluster ${ }^{10}$ which effectively manages and orchestrates docker containers. This allows us to scale microservices, running multiple instances and balancing their load automatically-even across physical servers (Figure 5, see $s 2$ ). We are further able to run multiple environments of the lingvis.io framework (middleware $\&$ frontend) in our cluster allowing our researchers to deploy a tailored version, for example, for a user evaluation. Kubernetes in combination with the reverse proxy trae$f i k^{11}$ automatically assigns a URL to the frontend and the middleware to make them accessible from anywhere in the world.

\section{Conclusion}

A demo of our framework is available under https://demo.lingvis.io/. Currently available visualizations with attendant NLP microservices are presented via the demo video or can be found under the "Visualizations" button. To the best of our knowledge, lingvis.io represents the first scaleable and modular web-based framework that combines NLP with visual analytics applications. Its unique contribution lies in combining these applications in a novel way on the one hand, but in separating NLP processing and visualizations on the operational level through an auto-configured pipeline on

\footnotetext{
${ }^{10}$ kubernetes.io

11 traefik.io
} 
the other hand. This enables developers to focus on the individual task at hand, rather than being distracted by needing to solve general NLP or visual analytics problems. As such, the framework is ideal for rapid prototyping and should serve as a productive base for more developments within LingVis, the interdisciplinary combination of, linguistics, NLP and visual analytics.

\section{References}

Miriam Butt, Annette Hautli-Janisz, and Verena Lyding. 2019. LingVis: Visual Analytics for Linguistics. CSLI lecture notes. CSLI Publications, to appear.

Mennatallah El-Assady, Valentin Gold, Carmela Acevedo, Christopher Collins, and Daniel Keim. 2016. ConToVi: Multi-Party Conversation Exploration using Topic-Space Views. Computer Graphics Forum, 35(3):431-440.

Mennatallah El-Assady, Annette Hautli-Janisz, Valentin Gold, Miriam Butt, Katharina Holzinger, and Daniel Keim. 2017a. Interactive Visual Analysis of Transcribed Multi-Party Discourse. In Proceedings of ACL 2017, System Demonstrations, pages 49-54, Stroudsburg, PA. ACL.

Mennatallah El-Assady, Rita Sevastjanova, Bela Gipp, Daniel Keim, and Christopher Collins. 2017b. NEREx: Named-Entity Relationship Exploration in Multi-Party Conversations. Computer Graphics Forum, 36(3):213-225.

Mennatallah El-Assady, Rita Sevastjanova, Daniel Keim, and Christopher Collins. 2018a. ThreadReconstructor: Modeling Reply-Chains to Untangle Conversational Text through Visual Analytics. Computer Graphics Forum, 37(3):351-365.

Mennatallah El-Assady, Rita Sevastjanova, Fabian Sperrle, Daniel Keim, and Christopher Collins. 2018b. Progressive Learning of Topic Modeling Parameters: A Visual Analytics Framework. IEEE Transactions on Visualization and Computer Graphics, 24(1):382-391.

Mennatallah El-Assady, Fabian Sperrle, Oliver Deussen, Daniel Keim, and Christopher Collins. 2019. Visual Analytics for Topic Model Optimization based on User-Steerable Speculative Execution. IEEE Trans. on Visualization and Computer Graphics, 25(1):374-384.

Mennatallah El-Assady, Fabian Sperrle, Rita Sevastjanova, Michael Sedlmair, and Daniel Keim. 2018c. LTMA: Layered Topic Matching for the Comparative Exploration, Evaluation, and Refinement of Topic Modeling Results. In International Symposium on Big Data Visual and Immersive Analytics (BDVA), pages 1-10.
Valentin Gold, Christian Rohrdantz, and Mennatallah El-Assady. 2015. Exploratory Text Analysis using Lexical Episode Plots. In Proc. of EuroVis., pages 85-89. The Eurographics Association.

Wolfgang Jentner, Mennatallah El-Assady, Bela Gipp, and Daniel A Keim. 2017. Feature Alignment for the Analysis of Verbatim Text Transcripts. In $E u$ roVis Workshop on Visual Analytics, EuroVA 2017, Barcelona, Spain, 12-13 June 2017, pages 13-17. Eurographics Association.

Wolfgang Jentner, Dominik Sacha, Florian Stoffel, Geoffrey P Ellis, Leishi Zhang, and Daniel A Keim. 2018. Making machine intelligence less scary for criminal analysts: reflections on designing a visual comparative case analysis tool. The Visual Computer, 34(9):1225-1241.

Daniela Oelke, David Spretke, Andreas Stoffel, and Daniel A. Keim. 2012. Visual readability analysis: How to make your writings easier to read. IEEE Transactions on Visualization and Computer Graphics, 18(5):662-674.

Dominik Sacha, Matthias Kraus, Jrgen Bernard, Michael Behrisch, Tobias Schreck, Yuki Asano, and Daniel A Keim. 2018. Somflow: Guided exploratory cluster analysis with self-organizing maps and analytic provenance. IEEE transactions on visualization and computer graphics, 24(1):120-130.

Christin Schätzle, Michael Hund, Frederik L Dennig, Miriam Butt, and Daniel A Keim. 2017. HistoBankVis: Detecting Language Change via Data Visualization. In Proceedings of the NoDaLiDa 2017 Workshop on Processing Historical Language, 133, pages 32-39. University of Konstanz, Germany.

Gerold Schneider, Mennatallah El-Assady, and Hans Martin Lehmann. 2017. Tools and Methods for Processing and Visualizing Large Corpora. Studies in Variation, Contacts and Change in English, 19.

Rita Sevastjanova, Fabian Beck, Basil Ell, Cagatay Turkay, Rafael Henkin, Miriam Butt, Daniel Keim, and Mennatallah El-Assady. 2018a. Going beyond Visualization: Verbalization as Complementary Medium to Explain Machine Learning Models.

Rita Sevastjanova, Mennatallah El-Assady, Annette Hautli-Janisz, Aikaterini-Lida Kalouli, Rebecca Kehlbeck, Oliver Deussen, Daniel Keim, and Miriam Butt. 2018b. Mixed-initiative active learning for generating linguistic insights in question classification. In Workshop on Data Systems for Interactive Analysis (DSIA) at IEEE VIS.

Fabian Sperrle, Jürgen Bernard, Michael Sedlmair, Daniel Keim, and Mennatallah El-Assady. 2018. Speculative Execution for Guided Visual Analytics. In Proc. of IEEE VIS Workshop on Machine Learning from User Interaction for Visualization and Analytics. 